\title{
Universal tessellations
}

\author{
DAVID SINGERMAN
}

\begin{abstract}
ARSTRACT. Ail maps of type $(m, n)$ are covered by a universal map $\mathscr{A}(m, n)$ which lies on one of the three simply connected Riemann surfaces; in fact $\overrightarrow{\mathscr{H}}(m, n)$ covers all maps of type $(r, s)$ where $r \mid m$ and $s \mid n$. In this paper we construct a tessellation $\hat{\mathscr{t}}$ which is universal for all maps on all surfaces. We also consider the tessellation $\mathscr{A}(\infty, 3)$ which covers all triangular maps. This coincides with the well-known Farey tessellation and we find many connections between $\hat{\boldsymbol{A}}(\infty, 3)$ and $\hat{\boldsymbol{A}}$.
\end{abstract}

\section{INTRODUCTION}

Let $\mathscr{P}$ be an orientable surface without boundary. A map $\mathscr{M}$ on $\mathscr{S}$ is a descomposition of $\mathscr{S}$ into simply-connected polygonal cells called faces. If $m$ is the least common multiple of the vertex valencies and $n$ is the least common multiple of the face valencies then we say that $\mathscr{M}$ has type $(m, n)$. In [5] it is shown that there is a regular map $\hat{\mathscr{M}}(m, n)$ on a simply-connected surface $\mathscr{U}$ whose vertices all have valency $m$ and whose faces all have valency $n$, and a subgroup $M$ of the automorphism group of $\hat{\mathscr{A}}(m, n)$ such that $\mathscr{U}$ $=\hat{\mathscr{A}}(m, n) / M$. This means that $\mathscr{S}=\mathscr{U} / M$ and the natural projection $p: \mathscr{U} \rightarrow \mathscr{S}$ carries $\mathscr{\mathscr { H }}(m, n)$ to $\mathscr{M}$. Thus $\hat{\mathscr{M}}(m, n)$ covers $\mathscr{M}$. (We shall describe $\hat{\mathscr{H}}(m, n)$ and its automorphism group in $\S 2$.) Hence $\hat{\mathscr{A}}(m, n)$ is universal for all maps of type $(m, n)$. In fact we shall see that it is universal for all maps of type $(r, s)$ where $r \mid m$ and $s \mid n$.

The aim of this paper is to describe a tessellation $\hat{\mathscr{A}}$ which is universal for all maps. $\hat{\mathscr{H}}$ will necessarily have vertices and faces of infinite valency and so we will not count it as a map with the usual definitions. (See [5], for example.) For this reason we use tessellation to describe these more general objects that we construct. We shall also construct a tessellation $\mathscr{M}(\infty, 3)$ which is universal for all triangular maps. This turns out to be an object

1980 Mathematics Subject Classification (1985 revision): -05C10, 20H05, 57M20, $10 \mathrm{D} 07$.

Editorial de la Universidad Complutense Madrid, 1988. 
which was first considered nearly a hundred years ago, [4]. It also has important relations to $\hat{\mathscr{H}}$.

The tessellations that we consider turn out to be related to the classical modular group PSL $(2, \mathbb{Z})$ and in a companion paper [6] we consider their algebraic and combinatorial properties in more detail.

Even though we are assuming that our surfaces are orientable and without boundary, we point out in $\$ 4$ that $\hat{\mathscr{H}}$ is universal for all maps on all surfaces. We find it noteworthy that though.$/ /$ covers all topological maps it is arithmetically defined; see Theorem 3.

\section{THE MAP $\hat{\mathscr{H}}(\mathrm{m}, \mathrm{n})$}

(See [5] for more details.) This map lies on the hyperbolic plane if $(1 / m)+(1 / n)<1 / 2$, the Euclidean plane if $(1 / m)+(1 / n)=1 / 2$, or the sphere if $(1 / m)+(1 / n)>1 / 2$. We start from a triangle $A B C$ with angles $\pi / 2, \pi / m, \pi / n$ lying in the appropriate plane. We shall call $A B C$ a basic triangle. We construct a map in which $A$ is an edge centre, $B$ is a vertex and $C$ is a face centre, as follows. Reflect the triangle in $A C$ to form a triangle $B B^{\prime} C$. We require $B B^{\prime}$ to be an edge of the map.

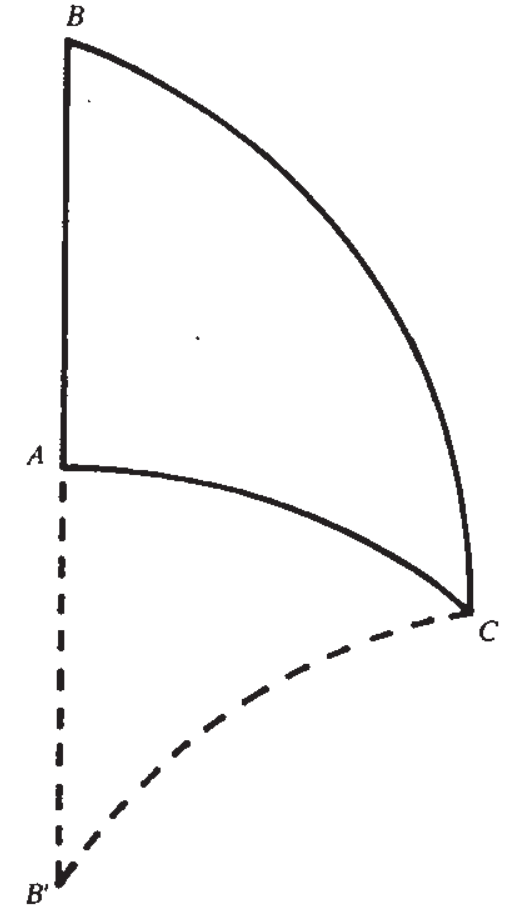

FIG. 1 
The angle at $C$ in $B B^{\prime} C$ is $2 \pi / n$ so that by rotating the triangle about $C$ we form $n$ triangles and hence a regular $n$-gon centred at $C$. We call this $n$-gon a principal face of the tessellation; in $\$ 3$ it is called a principal triangle. The $n$ gon is then one of the faces of the map $\hat{A}(m, n)$ all of whose vertices have valency $m$ and all of whose faces have valency $n$. This is the universal map of type $(m, n)$. For finite values of $m$ and $n$ it is very well known, for example $\mathscr{A}(3$, $6)$ is the honeycomb tessellation. Later, we will be interested in the cases where $m$ and $n$ can take the 'value' $\infty$.

The automorphism group of $\hat{\mathscr{M}}(m, n)$ is the triangle group $\Gamma(2, m, n)$ which has presentations

$$
\begin{aligned}
& g p<x, y, z \mid x^{2}=y^{m}=z^{n}=x y z=1> \\
& =g p<x, y \mid x^{2}=y^{m}=\left(y^{-1} x\right)^{n}=1>.
\end{aligned}
$$

This group is associated in two ways with the map $\hat{M}(m, n)$. Firstly, we consider $x, y, z$ to be rotations through $\pi, 2 \pi / m, 2 \pi / n$ about $A, B, C$ respectively. These rotations then generate a group with fundamental region $B B^{\prime} C$ which is isomorphic to $\Gamma(2, m, n)$. In this way $\Gamma(2, m, n)$ acts as a group of automorphisms of $\hat{\mathscr{M}}(m, n)$.

Another representation of $\Gamma(2, m, n)$ is as a permutation group of the darts as we now describe. When an edge $e$ of $\mathscr{A}(m, n)$ meets a vertex $v$ we draw an arrow along $e$ towards $v$. This arrow is called a dart of $\hat{\mathscr{A}}(m, n)$ and we let $\Omega(m, n)$ denote the set of darts of $\hat{M}(m, n)$. Each edge carries two darts and we define $\bar{x}$ to be the permutation of $\Omega(m, n)$ of order two which interchanges the darts of every edge. Thus $\bar{x}$ has a two-cycle for every edge of $\hat{\mathscr{M}}(m, n)$. Now following an anticlockwise orientation around a vertex $v$ gives us a cyclic permutation of the $m$ darts pointing towards $v$ and we define a permutation $\bar{y}$ which is a product of all such cyclic permutations. Thus $\bar{y}$ has an $m$-cycle for every vertex of $\hat{M}(m, n)$. We then find that $\bar{z}=\bar{y}^{-1} \bar{x}$ is a product of $n$-cycles, there being one such cycle for every face of $\hat{\mathscr{M}}(m, n)$. Now let $\Gamma(2, m, n)$ be the group generated by $\bar{x}$ and $\bar{y}$. We will show that $\bar{\Gamma}(2, m, n)$ is isomorphic to $\Gamma(2, m, n)$.

As $\Gamma(2, m, n)$ acts as a group of automorphisms of $\hat{\mathscr{M}}(m, n)$ there is an obvious action of $\Gamma(2, m, n)$ on $\Omega(m, n)$ which commutes with the action of $\tilde{\Gamma}(2, m, n)$. Now $\Gamma(2, m, n)$ clearly acts transitively on $\Omega(m, n)$ and as $\hat{\mathscr{A}}(m, n)$ is connected $\Gamma(2, m, n)$ also acts transitively on $\Omega(m, n)$ by proposition 2.2 of [5]. Also, by properties of discrete groups $\Gamma(2, m, n)$ also acts freely on $\Omega(m, n)$. As the actions of $\Gamma(2, m, n)$ and $\Gamma(2, m, n)$ commute it is easy to see that $\Gamma(2, m, n)$ also acts freely on $\Omega(m, n)$. Thus if $\alpha \in \Omega(m, n)$ and $\bar{g} \in \bar{\Gamma}(2, m, n)$ then there is a unique $g \in \Gamma(2, m, n)$ such that $\bar{g}(\alpha)=g(\alpha)$. Now

$$
\bar{g}_{1} \bar{g}_{2}(\alpha)=g_{1} \bar{g}_{2}(\alpha)=\bar{g}_{2} g_{1}(\alpha)=g_{2} g_{1}(\alpha)
$$


and thus the function $\Phi: \Gamma(2, m, n) \rightarrow \Gamma(2, m, n)$ given by $\Phi(\bar{g})=g^{-1}$ is an isomorphism.

We now let $\mathscr{M}$ be any map on an orientable surface $\mathscr{P}$. Then we form permutations $X, Y$ of the darts analogously to how we formed $x, y$ above. (We use the orientation of $\mathscr{S}$ to define the permutation $Y$.) If $\mathscr{A}$ has type $(r, s)$ where $r|m, s| n$, then

$$
X^{2}=Y^{r}=\left(Y^{-1} X\right)^{s}=1
$$

and so if $G=g p<X, Y>$ there is a homomorphism $\Theta: \Gamma(2, m, n) \rightarrow G$. If $\delta$ is a dart of $\mathscr{M}$ and if $G_{\delta}$ is the stabilizer of $\delta$ in $\mathrm{G}$, then $M=\Theta^{-1}\left(G_{\delta}\right)$ is a subgroup of $\Gamma(2, m, n)$. It is called a map subgroup of $\mathscr{M}$. As $M<\Gamma(2, m, n)$ it acts a group of automorphisms of $\mathscr{M}(m, n)$ and so we can form the quotient map $\hat{\mathscr{A}}(m, n) / M$. If $[\delta]_{M}$ is dart in $\hat{\mathscr{M}}(m, n) / M$ then $g \in \Gamma(2, m, n)$ acts by $[\delta]_{M} \rightarrow[g \delta]_{M}$. As $\Gamma(2, m, n)$ acts freely as a permutation group of the darts of $\mathscr{A}(m, n)$, the map subgroup $M$ is the stabilizer of the induced action of $\Gamma(2, m, n)$ on the darts $\hat{\mathscr{M}}(m, n) / M$. Thus $\mathscr{M}$ and $\hat{\mathscr{M}}(m, n) / M$ have the same map subgroup so by [1] or [5] we deduce that $\mathscr{\mathscr { M }}(m, n) / M$ is isomorphic to $\mathscr{M}$. (The concept of map subgroup and its use in the algebraic theory of maps first occurred in [5].)

Thus $\hat{\mathscr{A}}(m, n)$ covers all maps of type $(r, s)$ where $r \mid m$ and $s \mid n$. We now describe how to construct universal tessellations $\hat{\mathscr{M}}(m, n)$ where one or both of $m$ or $n$ can be $\infty$. We will see that it makes sense to write $k \mid \infty$ for all positive integers $k$. The idea behind our construction is that in hyperbolic geometry there are rotations of infinite order that generate discrete groups. We remind the reader how this occurs.

If we consider the Poincaré upper half-plane model $\mathbb{H}$ of hyperbolic geometry, then the sense-preserving isometries are the real Möbius transformations,

$$
z \rightarrow \frac{a z+b}{c z+d}, \quad a, b, c, d \in \mathbb{R}, \quad a d-b c=1 .
$$

The rotations are represented by the elliptic elements, those for which $|a+d|<2$. These are also characterised by having a single fixed point in $\mathbb{H}$, the centre of rotation. Transformations for which $|a+d|=2$ are called parabolic and have a single fixed point in $\mathbb{R} \cup\{\infty\}$. We can think of $\mathbb{R} \cup\{\infty\}$ as being the line at infinity in the hyperbolic plane so parabolic elements are rotations about 'a point at infinity'. For this reason, parabolic elements were called limit rotations in some older books. They can be thought of as elliptic elements of infinite order. 
Every parabolic element is conjugate to $z \rightarrow z \pm 1$ and so generates an infinite cyclic discrete group. A triangle group $\Gamma(2, \infty, n)$ has a presentation

$$
<x, y, z \mid x^{2}=z^{n}=x y z=1>\text {. }
$$

The 'relation' $y^{\infty}=1$ is regarded as being vacuous but we can read it as ' $y$ is parabolic'. Note that there is a homomorphism from $\Gamma(2, \infty, n)$ to $\Gamma(2, m, n)$ for all integers $n$, so that any map subgroup in $\Gamma(2, m, n)$ can be pulled back to $\Gamma(2, \infty, n)$. The statement $k \mid \infty$ is explained by the existence of a homomorphism from the infinite (parabolic) cyclic group onto the finite (elliptic) cyclic group of order $k$.

\section{THE UNIVERSAL TRIANGULAR TESSELLATION}

Before we describe the universal tessellation, we discuss the universal triangular tessellation, which turns out to be a more familiar object. The universal triangular tessellation is denoted by $\hat{\mathscr{H}}(\infty, 3)$ and it is constructed in the same way as we formed $\mathscr{M}(m, n)$. Thus we start with a triangle with angles $\pi / 2, \pi / 3, \pi / \infty(=0)$. As $\Gamma(2, \infty, 3)$ is isomorphic to $\Gamma(2,3, \infty)$ which, in turn, is isomorphic to $\operatorname{PSL}(2, \mathbb{Z})$, (the modular group), we choose for our basic triangle half of the standard fundamental domain for the modular group. This has vertices $i, \rho=(1+i \sqrt{3}) / 2$ and $\infty$.

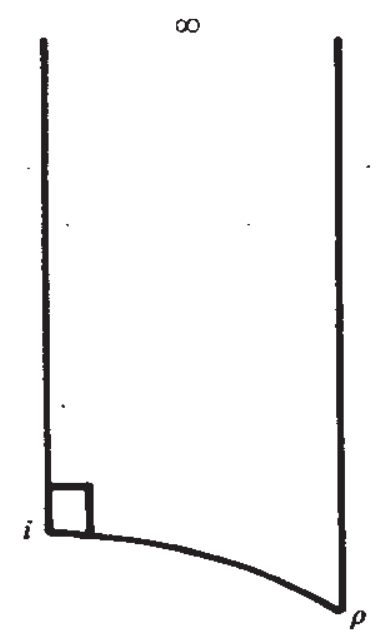

FIG. 2

Following the procedure before, we construct a tessellation in which $\infty$ is a vertex, $i$ is an edge-centre and $\rho$ is a face-centre. The rotation of order 3 about $\rho$ is $z \rightarrow-1 /(z-1)$ which maps $(0, \infty, \rho)$ to $(1,0, \rho)$ and then to $(\infty, 1$, $\rho$ ). Thus a principal triangle of the tessellation has vertices 0,1 , and $\infty$. 


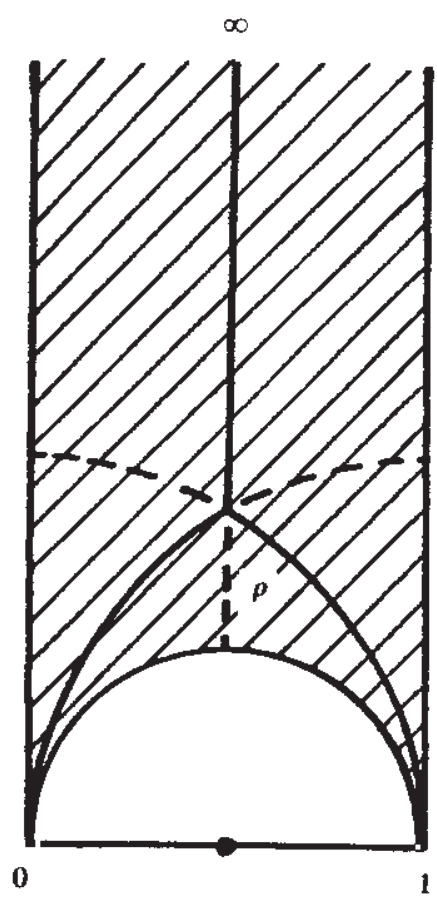

FIG. 3

Now an edge of the tessellation is $(\infty, 0)$, (this is the directed edge going from $\infty$ to 0 ), and if we apply the modular transformation

$$
z \rightarrow \frac{a z+b}{c z+\bar{d}} \quad a, b, c, d \in \mathbb{Z} \quad a d-b c=1
$$

then this is mapped to the edge $(a / c, b / d)$. Because of the condition $a d-b c=1$, these fractions are in their lowest terms. Conversely, if $a / c$ and $b / d$ are fractions in their lowest terms with $a d-b c=1$, then they are joined by an edge of the tessellation. (We regard $\infty=1 / 0$.) Thus the universal triangular tessellation has the extended rationals $Q^{*}=Q \cup\{\infty\}$ as vertex set and two vertices $a / c$ and $b / d$ are joined by an edge if and only if $a d-b c= \pm 1$. We draw the edges as geodesics in the hyperbolic geometry of the Poincare halfplane, that is as semi-circles or lines perpendicular to $\mathbb{R}$. The resulting tessellation is often called the Farey tessellation $\mathbb{F}$. Thus $\mathbb{F}=\hat{\mathscr{A}}(\infty, 3)$. It seems to have first been introduced by A. Hurwitz in 1894 [4], with applications to number theory in mind. It is discussed in more detail in the companion paper [6], where the elementary result that the underlying graph is connected is proved. 


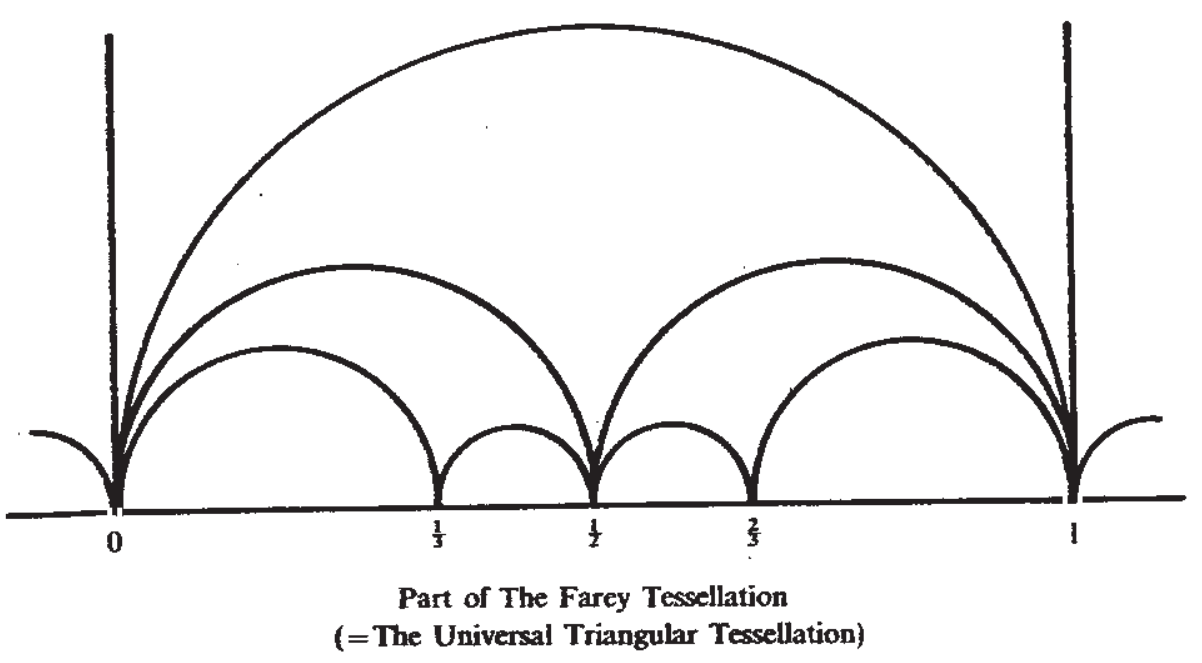

FIG. 4

Now if $M$ is a subgroup of finite index in $\Gamma(2, \infty, 3)$ then $\left(H \cup Q^{*}\right) / M$ is a surface (as shown in books on the modular group, for example [7]), and so the Farey tessellation projects to a triangular tessellation on the surface. As $M$ has finite index, the surface is compact; furthermore, for the same reason, the vertices have finite valency, so that this tessellation is a triangular map. We can obtain triangular maps on non-compact surfaces by replacing finite index with the weaker condition that all parabolic elements of $\Gamma(2, \infty, 3)$ have finite order modulo $M$. We now show that all triangular maps can be obtained in this way.

Theorem 1. Let $T$ be a triangular map. Then there is a subgroup $M \leqslant \Gamma(2, \infty, 3)$ such that $\mathbb{F} / M \simeq T$.

Proof. Let $X, Y$ be the permutations of the darts as described in $\$ 2$. As $T$ is triangular, $\left(Y^{-1} X\right)^{3}=1$ so that there is a homomorphism

$\Theta: \Gamma(2, \infty, 3) \rightarrow G=g p<X, Y>$. Again, $M=\Theta^{-1}\left(G_{\delta}\right)$ can be regarded as a map subgroup for $T$. Now, $\Gamma(2, \infty, 3)$ acts as a group of automorphisms of $\mathbb{F}$ and we now observe that its action on the darts of $\mathbb{F}$ is regular, that is transitive and free. To see this note that a dart of $F$ can be represented as an ordered pair of rationals $(a / c, b / d)$, this being the dart pointing along the edge from $a / c$ to $b / d$. This is the image of the principal dart $(1 / 0,0 / 1)$ under the modular transformation (1). To show that the action is free we just note that only the identity fixes the dart $(1 / 0,0 / 1)$.

As $\Gamma(2, \infty, 3)$ acts regularly on the darts of $\mathbb{F}$ we see, as in $\$ 2$, that the map subgroup of $F / M$ is $M$. Thus $\mathbb{F} / M$ and $T$ have the same map subgroup and so $\mathbb{F} / M \simeq T$. 
In this sense then, $\mathbb{F}$ can indeed be regarded as the universal triangular tessellation. All triangular tessellations are quotients of $\mathbb{F}$ and so $\mathbb{F}$ covers all triangular maps.

\section{THE UNIVERSAL TESSELLATION}

By considering the groups $\Gamma(2, \infty, n)$ for $n>3$, we can construct universal $n$-gonal tessellations. The groups $\Gamma(2, \infty, n) \simeq \Gamma(2, n, \infty)$ are Hecke groups and are rather more complicated than the modular group. (The matrix entries lie in the field $Q(\cos \pi / n)$. We shall be more interested in jumping straight to $\Gamma(2, \infty, \infty)$ so that we can construct the universal tessellation. Luckily, this group is isomorphic to a subgroup of the modular group.

Let $\Gamma_{0}(2)$ be the subgroup of the modular group consisting of all entries in (1) for which $c$ is even. It is well-known that $\Gamma_{0}(2)$ is a non-normal subgroup * of index 3 in the modular group.

Let $T$ be the hyperbolic triangle with vertices $(1+i) / 2,0$ and $\infty$. If we reflect $T$ in the imaginary axis, then we obtain the hyperbolic quadrilateral $Q$ bounded by the circles $|z-(1 / 2)|=1 / 2,|z+(1 / 2)|=1 / 2$ and the lines $\operatorname{Re}(z)=$ $\pm 1 / 2$. By using isometric circles, it is easily seen that $Q$ is a fundamental domain for $\Gamma_{0}(2)$, (see [3], for example) and as $T$ has angles $\pi / 2,0$ and 0 it follows that $\Gamma_{0}(2)$ is isomorphic to $\Gamma(2, \infty, \infty)$. See Fig. 5. (As the referee has pointed out, it is more consistent with the previous paragraphs to use a triangular fundamental region bounded by the hyperbolic lines $\operatorname{Re}(z)=0$, $\operatorname{Re}(z)=1 / 2,|z-1 / 4|=1 / 4$.)

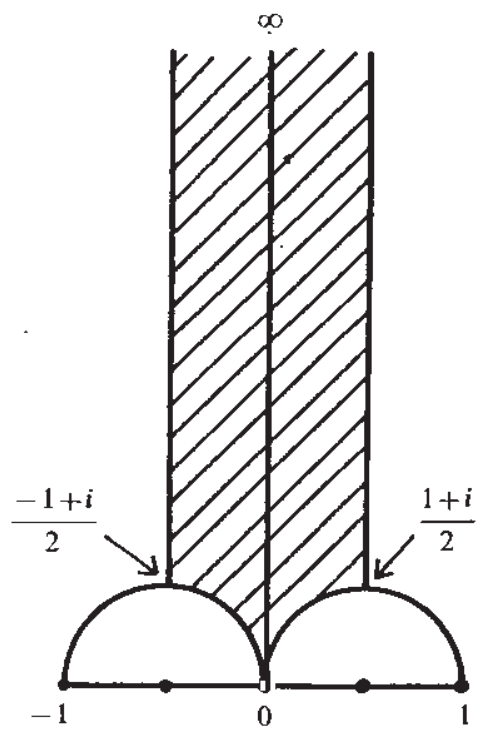

FIG. 5 
We now construct the tessellation $\hat{\mathscr{A}}=\hat{\mathscr{M}}(\infty, \infty)$, (which we show later is a universal tessellation). We regard $T$ as a basic triangle, 0 as a face centre, and $(1+i) / 2$ as an edge centre. The parabolic subgroup fixing 0 in $\Gamma_{0}(2)$ is the cyclic subgroup generated by

$$
A(z)=\frac{z}{2 z+1}
$$

so a principal face of the tessellation is

$$
\bigcup_{k=-\infty}^{\infty} A^{k}(Q)
$$

Theorem 2. Let $m$ be an integer not equal to 0 or -1 , and let $S_{m}$ denote the circle with real centre which passes through $1 / 2 m$ and $1 /(2 m+2)$. Then

$\bigcup_{\bigcup}^{\infty} A^{k}(Q)$ is the region $P$ in $\mathbb{H}$ bounded by the circles $S_{m}(m=1, \pm 2, \pm 3, \ldots)$ and the lines $\operatorname{Re}(z)= \pm 1 / 2$. (See Fig. 6.)

Proof. We note that

$$
A^{k}(z)=z /(2 k z+1)
$$

so that $A^{k}(\infty)=1 / 2 k, A^{k}(1 / 2)=1 /(2 k+2), A^{k}(0)=0, A^{k}(-1 / 2)=1 /(2 k-2)$.

Thus the images of the four vertices of $Q$ lie on the boundary of $P$. As $Q$ is hyperbolically convex, $A^{k}(Q) \subseteq P$ so that

$$
\bigcup_{k=-\infty}^{\infty} A^{k}(Q) \subseteq P \text {. }
$$

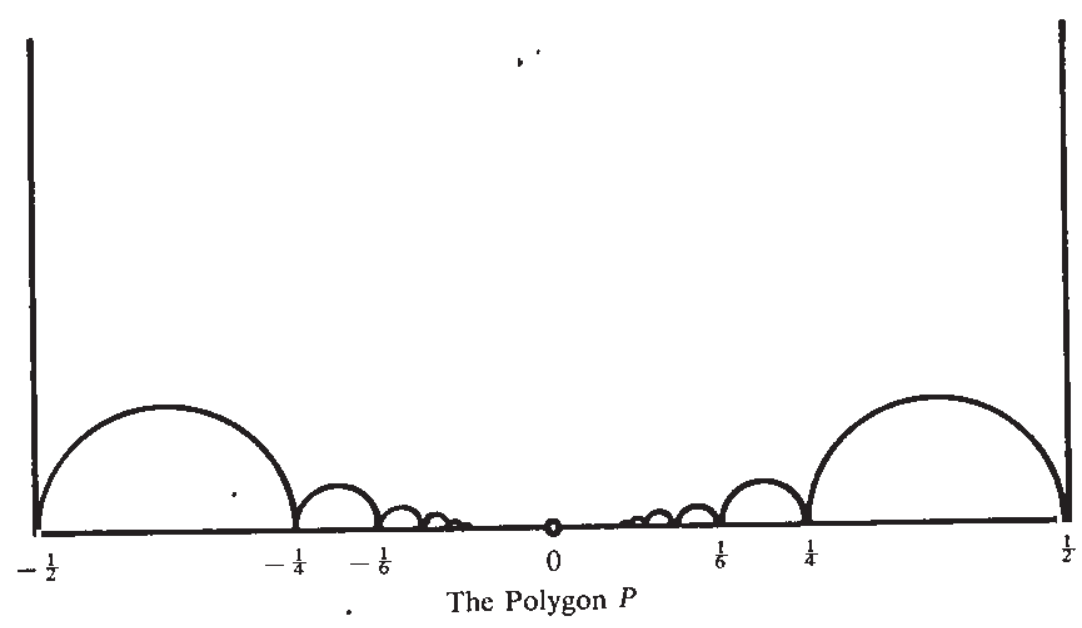

FIG. 6 
Now $A((-1+i) / 2=(1+i) / 2, A(\infty)=1 / 2$, so that the vertical line segment from $(-1+i) / 2$ to $\infty$ is mapped to the vertical line segment from $(1+i) / 2$ to $1 / 2$. Thus the vertical line segment $L$ from $1 / 2$ to $\infty$ bounds the polygon $Q^{\prime}=Q \cup A(Q)$. The image of $L$ under $A^{k},(k=1, \pm 2, \pm 3, \ldots)$ is the upperhalf of the circle $S_{k}$. Thus these semi-circles $S_{k}$ also bound $\bigcup^{\infty} A^{k}(Q)$. The image of $L$ under $A^{-1}$ is the vertical line segment from $-1 / 2$ to $\infty$. Thus

$$
\bigcup_{k=-\infty}^{\infty} A^{k}(Q)=P
$$

We consider $P$ as being an infinite-sided polygon with vertices $1 / 2 k$ $(k \in Z-\{0\})$ and $\infty$. We do not consider 0 as a vertex. However, as 0 is fixed by the parabolic (or limit rotation) $A$, which cyclically permutes the vertices of $P$, we regard 0 as a face-centre of $P$.

The tessellation $\hat{\mathscr{H}}$ then, is the union of all images of $P$ under $\Gamma_{0}(2)$. As $P$ is a union of $\Gamma_{0}(2)$ - images of the fundamental domain $Q, \hat{A}$ is indeed a tessellation of the upper half-plane.

Before we actually prove the universal property of $\hat{M}$, we note some of its properties, particularly of its underlying graph.

\section{Part of The Universal Tessellation $\hat{\mathscr{A}}$}

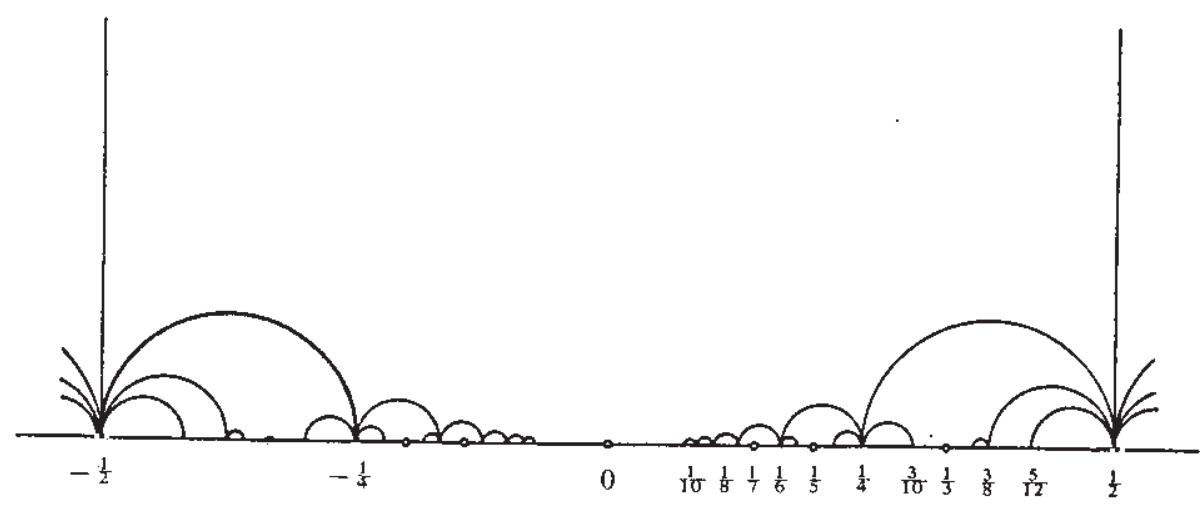

FIG. 7

Theorem 3. (a) The vertices of $\hat{\mathscr{A}}$ are the rationals with even denominator, including $1 / 0=\infty$.

(b) The faces centre of $\hat{\mathscr{A}}$ are the rationals with odd denominator.

(c) Two vertices $a / b$, c/d are joined by an edge if and only if $a d-b c= \pm z$.

(d) $\Gamma_{0}(2)$ acts regularly on the directed edges of $\hat{\mathcal{M}}$. 
(e) Two rationals $x / y, u / v$ are face centres of adjacent faces if and only if $x v-y u= \pm 1$. \pm 1 .

(f) $A$ vertex $a / b$ belongs to a face with centre $x / y$ if and only if $a y-b x=$

Proof. (a) The vertices of $P$ are the images of $\infty$ under $A^{k} \in \Gamma_{0}$ (2). Hence the vertices of $\hat{A}$ are the $\Gamma_{0}(2)$-images of $\infty=1 / 0$. Let

$$
T(z)=(p z+q) /(2 r z+s) \in \Gamma_{0}(2) .
$$

Then $T(\infty)=p / 2 r$ which has even denominator. Conversely, given the rational $p / 2 r$ with $(p, 2 r)=1$ we can find integers $q, s$ such that $p s-2 q r=1$. Thus there exists a transformation $T \in \Gamma_{0}(2)$ with $T(\infty)=p / 2 r$.

(b) The face-centres are the $\Gamma_{0}(2)$-images of 0 . The image of 0 under $T$ above is $q / s$ and $s$ is odd as $p s-2 q r=1$.

(c) Similarly, the edges of $\hat{M}$ are the $\Gamma_{0}(2)$-images of the edge $(1 / 0,1 / 2)$ from $\infty$ to $1 / 2$. The image of this edge under $T$ is

$$
(p / 2 r,(p+2 q) /(2 r+2 s))
$$

and $p(2 r+2 s)-2 r(p+2 q)=2(s p-2 r q)=2$.

Conversely, if $a d-b c=2$, and $b, d$ are even, $a, c$ are odd then

$$
z \rightarrow \frac{a z+(c-a) / 2}{\overline{b z+(d-b) / 2}} \in \Gamma_{0}(2)
$$

and maps $(1 / 0,1 / 2)$ to $(a / b, c / d)$. If $a d-b c=-2$, then we just change the direction of the edge.

(d) In $(c)$ we showed that $\Gamma_{0}(2)$ acts transitively on the directed edges. However, if $T \in \Gamma_{0}(2)$ fixes both $1 / 0$ and $1 / 2$ then $T$ is the identity, so the action is regular.

(e) We note that $1=1 / 1$ is a face-centre of a face adjacent to $P$. Hence all such faces have centres $A^{k}(1)$. Thus if $x / y$ and $u / v$ are adjacent face-centres, then for some $S \in \Gamma_{0}(2), S(0 / 1)=x / y, S(1 / 1)=u / v$. As $0.1-1.1=-1$, we find that $x v-y u=-1$. As above, if $x v-y u=-1$ then we can find an $S \in \Gamma_{n}(2)$ with $S(0 / 1)=x / y, S(1 / 1)=u / v$, so that $x / y, u / v$ are adjacent face-centres.

(f) The vertices of $P$ are $1 / 2 k(k \in \mathbb{Z})$ and the face-centre is $0 / 1$. As $1.1-2 k .0=1$, the result follows as in $(e)$.

Corollary 1. $\hat{\mathscr{A}}$ is isomorphic to the subtessellation of the Farey tessellation whose vertices have odd numerator. 
Proof. By parts $(a)$ and $(c)$ of the theorem, the function which takes $a / 2 b$ to $a / b$ is the required isomorphism.

Corollary 2. The dual of $\hat{M}$ has vertices $x / y$ ( $y$ odd) with $x / y$ joined to $u / v$ by an edge if and only if $x v-y u= \pm 1$. Thus the dual of $\hat{\vec{H}}$ is the subtessellation of the Farey tessellation whose vertices have odd denominator.

Proof. This follows from $(e)$ above.

Corollary 3. If we triangulate $\hat{A}$ by joining vertice to face centres by edges, then we get the Farey tessellation.

Proof. This follows from $(f)$.

Remark. It is shown in [6] that the underlying graph of $\hat{M}$ is a suborbital graph for the modular group and is topologically a tree.

We now prove the universal property of the tessellation $\mathscr{A}$. We just follow the procedure in $\$ 3$ for triangular tessellations.

If $M<\Gamma_{0}(2)$ then $\left(\mathbb{H} \cup Q^{*}\right) / M$ is a surface and if all the parabolics of $M$ have finite order $\bmod M$ then.$\hat{M}$ projects to a map on the surface, for then all faces and vertices have finite valency. For example, if $A$ (the parabolic generator fixing 0 ) has order $k \bmod M$, then $P$ projects to a $k$-gon on the surface. Furthermore, we have the following result.

Theorem 4. Let $\mathscr{M}$ be a map on a surface $\mathscr{P}$. Then there is a subgroup $M<\Gamma_{0}(2)$ such that $\hat{M} / M \simeq \mathscr{M}$.

Proof. Let $X, Y$ be the permutations of the darts as described in $\S 2$. Then $X^{2}=1$ and because $\Gamma_{0}(2) \simeq \Gamma(2, \infty, \infty)$ there is a homomorphism $\phi: \Gamma_{0}(2) \rightarrow G=g p<X, Y>$. As $\Gamma_{0}(2)$ acts regularly on the darts of $\hat{\mathscr{M}}$ by theorem $3(d)$, we can show that $\mathscr{M} \simeq \mathscr{M} / M$ where $M=\phi^{-1}\left(G_{\delta}\right)$ in the same way as in theorem 1.

Even though we have been assuming that our surfaces are orientable and without boundary, the above result does apply to more general surfaces. For let $\mathscr{H}$ be a map on a surface $\mathscr{P}$ which is either non-orientable, or has boundary, or both. Then, following [1], we can form the canonical double $\mathscr{M}^{d}$ of $\mathscr{M}$, which lies on the canonical double $\mathscr{S}^{d}$ of $\mathscr{S}$. The surface $\mathscr{S}^{d}$ is orientable and without boundary so $\mathscr{P}^{d}$ is covered by $\mathrm{H}_{\text {. }}$. Also, $\mathscr{P}^{d}$ covers $\mathscr{P}$ in the more general sense that we allow folding if $\mathscr{P}$ has boundary. Hence in this more general sense $\hat{\mathscr{H}}$ covers $\mathscr{M}$. (In fact, $\mathscr{M}$ will have a map subgroup lying in the reflection group containing $\Gamma_{0}(2)$ with index 2 .)

\section{BIPARTITE MAPS}

By theorem 3(b), the face-centres of $\hat{\mathscr{M}}$ are rationals with odd denominator. By theorem 3(e), if two faces are adjacent then the numerators 
of the face-centres cannot have the same parity. Thus we can two-colour the faces of $\hat{\mathscr{M}}$ according to the parity of the numerator. Now it is easy to see that the subgroup of $\Gamma_{0}(2)$ which preserves the set of rationals of the form odd/odd, and hence those of the form even/odd, is $\Gamma(2)$, the principal congruence subgroup of level 2 ; this is defined by

$$
z \rightarrow \frac{a z \pm \underline{b}}{c z+d}, \quad a, b, c, d \in \mathbb{Z}, \quad a d-b c=1, \quad\left(\begin{array}{ll}
a & b \\
c & d
\end{array}\right) \equiv\left(\begin{array}{ll}
1 & 0 \\
0 & 1
\end{array}\right) \bmod 2 .
$$

This has index 2 in $\Gamma_{0}(2)$ and is isomorphic to the triangle group $\Gamma(\infty, \infty, \infty)$.

If $M<\Gamma(2)$, then as $M$ preserves the two-colouring of $\hat{M}, \hat{\mathscr{M}} / M$ is bipartite, and all bipartite maps occur in this way.

Now by a result of Walsh [8], bipartite maps are in one-to-one correspondence with hypermaps. It is shown in [2] that hypermaps correspond to subgroups of $\Gamma(\infty, \infty, \infty)$ in the same way that maps correspond to subgroups of $\Gamma(2, \infty, \infty)$. This section can thus be viewed as an interpretation of Walshes theorem in terms of the modular group.

I would like to thank Gareth Jones for his helpful criticism and advice and also Rose Cassell for drawing the diagrams.

The material in this paper formed the subject of one of a series of talks I gave on maps in Madrid during July 1987. I would like to thank Universidad a Distancia for their hospitality and financial support.

\section{References}

1. R. P. BRYANT and D. Singerman, Foundations of the theory of maps on surfaces with boundary, Quart. J. Math. Oxford (2), 36 (1985), 17-41.

2. D. CORN and D. SINGERMAN, Regular hypermaps, European Jnl. of combinatorics 9 (1988), 337-351.

3. L. R. FORD, Automorphic Functions (2nd ed.) Chlesea, New York (1951). .

4. A. Hurwirz, Über die Reduktion der binären quadratischen formen, Math. Ann. $45(1894), 85-117$.

5. G. A. Jones and D. Singerman, Theory of maps on orientable surfaces, Proc. London Math. Soc. (3), 37 (1978), 273-307.

6. G. A. JonES, D. SINGERMAN and K. WiCKS, The modular group and generalized Farey graphs; in preparation.

7. B. SCHOENEBERG, Elliptic modular functions, Springer-Verlag, Berlin, Heidelberg. New York.

8. T, R. S. WALSH, Hypermaps versus bipartite maps, J. Combin. Theory Ser. B 18 (1973), 155-163. 